\title{
Image Similarity Learning through Rankboost Mechanism Based On Keywords and Content Queries
}

\author{
Veena Sharanya. $\mathrm{M}^{1}$, Evelyn Brindha. $\mathrm{V}^{2}$ \\ ${ }^{1} P G$ Scholar, Department of CSE, Bannari Amman Institution of Technology, India \\ ${ }^{2}$ Associate Professor, Department of CSE, Bannari Amman Institution of Technology, India.
}

\begin{abstract}
Different types of search engines are used to search image and text contents. Two types of image search methods are available in the Internet. They are query keyword based model and content based image retrieval models. Text query strings are used in the textual image retrieval model. Content based image retrieval (CBIR) model uses the visual information of the images. Image search methods use the text annotation and image visual features. Google image search and Bing image search engines are used to fetch images from the web.

Image query string is used to search image on Internet. One click query image selection method is used to submit user intention for image retrieval. Content based image re-ranking is performed with visual and textual similarity metrics. Adaptive Weight Schema is used for the similarity analysis. Feature weight learning algorithm is applied to estimate feature weights for the images and its category. Query is expanded with keyword and visual information.

Rank boost framework algorithm is enhanced to rank images with photographic quality. Content similarity and visual quality factors are used for the re-ranking process. In this paper, we propose an image indexing and retrieval using speech annotations based on a predefined structured syntax. To improve the retrieval effectively, $N$-best lists for index generation is used .so, a query expansion technique is explored to enhance the query terms. All this process is automatic, without extra effort from the user. This is critically important in web-based image search engine for any commercial, where the user interface has to be extremely simple.
\end{abstract}

Keywords: intension, image retrieval, adaptive similarity, keyword expansion, image reranking, Speech recognition, multimedia information retrieval.

\section{Introduction}

Digital photography, public networks of public networks and cheap storage have led to a rapid increase in the use of digital images in many application areas, such as publishing and the commerce, military, media, education, and the World Wide Web. The need to manage these images and locate target images in response to user search queries has become a significant problem One way to solve this problem would be describing the image by keywords and content query. Many commercial Internet scale used only text as queries used for image search engines. Users type keywords query in the hope of finding a certain type of images. The keywords extracted from the surrounding text, search engine returns thousands of images ranked. It is known that textbased image search suffers from the ambiguity of query keywords.

A novel Internet based image search approach. The user required giving only one click on a query image and images from a pool retrieved by text-based search are reranked based on their visual and textual similarities to the query image.

\section{Related Works}

When the retrieved information is a collection of images called Image Retrieval. The origins on Image Retrieval can be traced back to when a conference on Database Techniques for Pictorial Applications [3]. Then the application potential of image database management techniques has attracted the attention of researchers. Early techniques were not based on visual features but on the textual annotation of images. However, this purely text-based approach posed two significant limitations in the retrieval of images [2]. The first limitation was related to the volume of the database. Manual annotation was such a cumbersome and expensive task that it could not be applied to large image databases. The second limitation which affected the performance of the system was that the description of the images was found to be a highly subjective task that could generate different text labels to the same image.

A rank learning algorithm called Imbalanced RankBoost by integrating the principles of RankBoost with an iterative thresholding scheme [4]. In addition to iteratively selecting and combining weak ranking features, the proposed approach provides a more efficient ranking process by automatically identifying a cutoff threshold in every run and truncating model computation. 
Similarity learning has an interesting reciprocal relation with classification. On one hand, pairwise similarity can be used in classification algorithms like nearest neighbors or kernel methods[5]. On the other hand, when objects can be classified into classes. The inferred labels induce a notion of similarity across object pairs. Similarity learning assumes a form of supervision that is weaker than in classification and no labels are provided. To learn a class-independent similarity measure with no need for class labels done using a design of OASIS.

\section{Feature Extraction Techniques}

Visual feature extraction is the basis of any content-based image retrieval technique and include both text-based features are keywords, speech annotations and visual feature extraction are color, texture, shape, etc. The scope of visual feature, the features can be further classified into a low-level features and high-level features.

\subsection{Color}

The color feature widely used visual features in image retrieval. Relatively robust to background complication and independent of image size and orientation. In image retrieval, the color histogram is the most commonly used color feature representation. One of the main aspects of color feature extraction is the choice of a color space. It is a multidimensional space in which the different dimensions represent the different components of color and is a three dimensional. An example of a color space is RGB, to each pixel assigned as the three element vector giving the color intensities of the three primary colors (green red and blue). The CIE color spaces represent, the three characteristics that best characterize color perceptually are hue, lightness, and saturation Because of the calculation complexities, the CIE color spaces are inconvenient of the transformation to and from the RGB color space. HSV color space is also a nonlinear transformation of the RGB. The HSV color space is approximately perceptually uniform to extract color features.

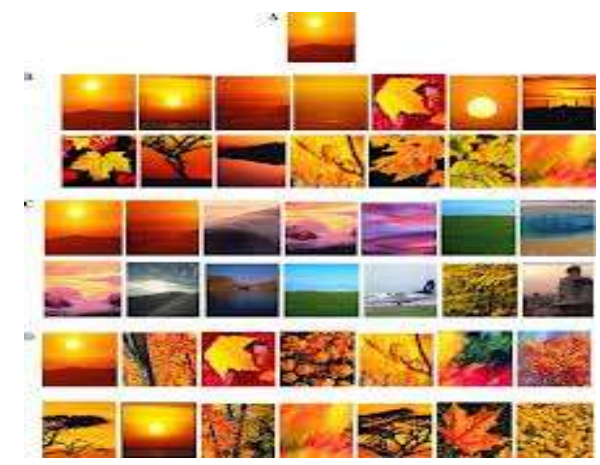

Fig 3.1.1: Image Retrieval Based On Color

\subsection{Texture}

Identify three features as being important in human texture perception are complexity, repetition and orientation. The properties contained in visual patterns that have homogeneity that do not result from the presence of only a single color or intensity. It is an innate property of virtually all surfaces, including trees, clouds, hair, bricks and fabric. It contains information about the structural arrangement surfaces and their surrounding environment to their relationships.

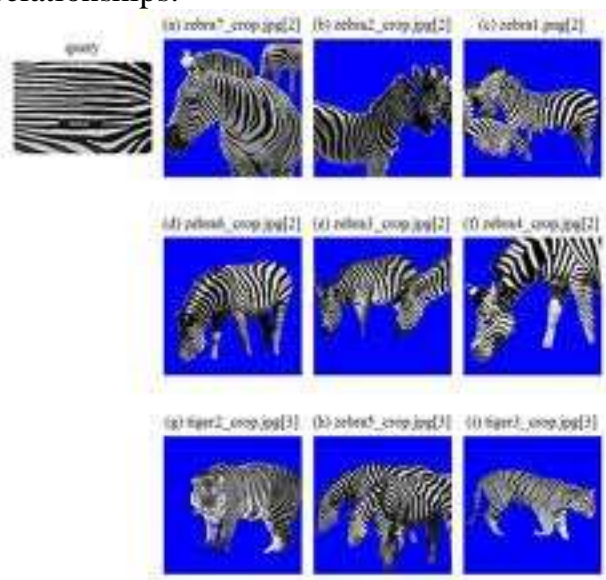

Fig 3.2.1: Image Retrieval Based On Texture 


\subsection{Shape:}

Shape is a visual feature and it is one of the primitive features for image content description. Queries for shapes are generally achieved by selecting an example image provided by the system or by having the user sketch a shape. The mechanisms used for shape retrieval include identification of features such as boundaries, lines, aspect ratio, and circularity, and by identifying areas of stability via region growing and edge detection. Of particular concern has been the problem of dealing with images having overlapping or touching shapes. Several problems remain including retrieval of features based on location within an image, the extension of 2-D features to 3-D, and appropriate segmentation of video images. Although research in higher order CBIR is underway, current systems are not capable of retrieving all instances of horses based on the shape, color, or texture of a single instance of a horse in a query. Although shape, color and texture are undoubtedly important visual features for image representation, and how best to implement these attributes for image retrieval. The technology for content-based image retrieval is still in its infancy.

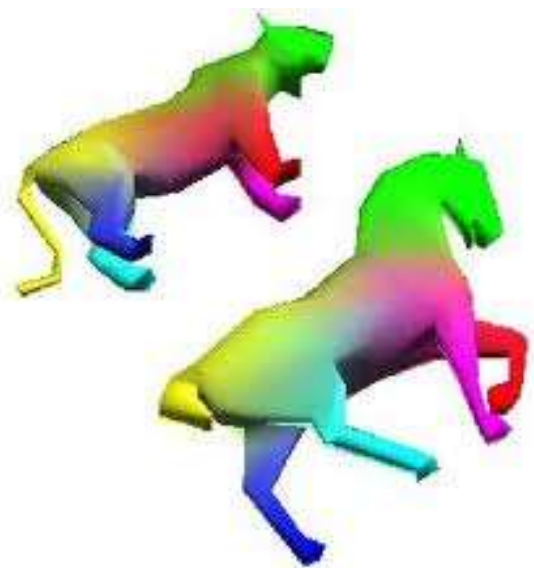

Fig 3.3.1: Image Retrieval Based On Shape

\section{Keyword And Content Queries For Retrieval}

A novel Internet based image search approach [1]. It requires the user to click on one query image with minimum effort and images from a pool retrieved by text-based search are reranked based on both visual and textual content.

To capture the users intention images from one-click query image in four steps:

1) The query image is categorized into one of the predefined adaptive weight categories which reflect users' search intention at a coarse level. A specific weight schema is used to combine visual features adaptive to this kind of image to better rerank the text based search result.

2) In the visual content of the query image selected by the user and through image clustering, query keywords are expanded to capture user intention.

3) To enlarge the image pool using expanded keywords to contain more relevant images.

4) keyword Expanded are used to expand the query image to multiple positive visual examples from which new query specific visual and textual similarity metrics are learned to further improve content-based image reranking.

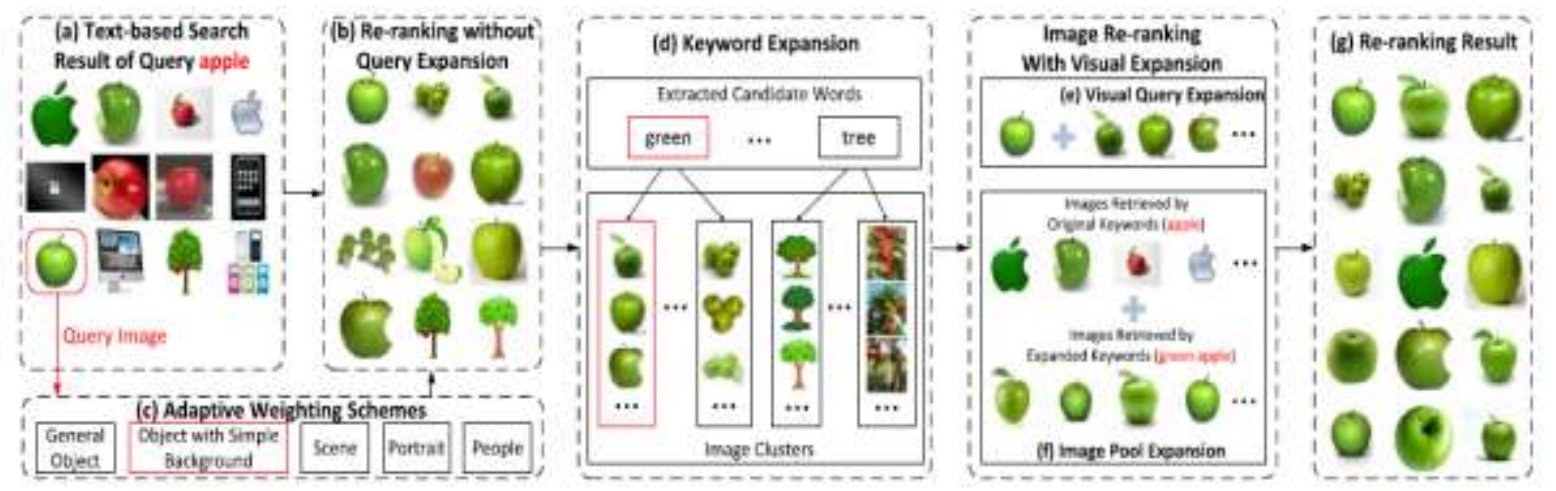

Fig 3.1 Image Retrieval Based On Rank Boost Algorithm 
a) Text-Based Search

The user first submits query keywords $\mathrm{q}$ as an input. Then an image is retrieved from a pool of image by textbased search. Then the user is asked to select a query image from the image pool.

b) Re-Ranking Without Query Expansion

Images in the pool are reranked based on their visual similarities to the query image and the similarities are computed using the weight schema specified by the category to combine visual features.

c) Adaptive weighted schemas

The query image is classified as one of the predefined adaptive weight categories. Human can easily categorize images into high-level semantic classes such as scene, object or people. And then observed that images inside these categories usually agree on the relative importance of features for similarity calculations. The query images into several typical categories, adaptively adjust feature weights are within each category.

d) Keyword Expansion

In the keyword expansion words are extracted from the textual descriptions (such as image file names and surrounding texts in the $\mathrm{html}$ pages) of the top $\mathrm{k}$ images most similar to the query image. To save computational cost, only the top $\mathrm{m}$ words are reserved as candidates for further processing because the initial image reranking result is still ambiguous and noisy, the top k images may have a large diversity of semantic meanings and cannot be used as visual query expansion[6],[7].

In this approach, reliable keyword expansions are found through further image clustering. For each candidate word wi, it find out all the images containing wi and group them into different clusters $\{\mathrm{ci}, 1, \mathrm{ci}, 2, \ldots$, ci,ti $\}$ based on visual content. The same candidate word with images may have a large diversity in visual content. Assign image to the same cluster have higher semantic consistency since they have high visual similarity to one another and contain the same candidate word.

e) Visual Query Expansion

The clusters contains of different candidate words, cluster ci,j with the largest visual similarity to the query image is selected as visual query expansion and its corresponding word wi is selected to form keyword expansion.

$$
\mathrm{q}^{\prime}=\mathrm{q}+\mathrm{wi}
$$

A query specific visual similarity metric and a query specific textual similarity metric are learned from both the query image and the visual query expansion.

f) Image Pool Expansion

The image pool is enlarged through combining the original image pool retrieved by the query keywords $\mathrm{q}$ provided by the user and an additional image pool retrieved by the expanded keywords q0. Images in the enlarged pool are reranked using the learned query-specific visual and textual similarity metrics.

g) Re-Ranking Result

The image cluster size is selected as visual query expansion and its similarity to the query image indicate the confidence that the expansion captures the user's search intention images from the pool.

\section{Conclusion}

A novel Internet image search approach which only requires one-click user feedback. Intention specific weight schema is to combine visual features and to compute visual similarity adaptive to image queries. Without adding human feedback, integration of textual and visual expansions is used to capture user intention. To enlarge the image pool to include more relevant images through expanded keyword used to extend the images.

This framework makes it possible for industrial scale image search by both text and visual content image. In the proposed new image reranking framework consists of multiple steps, can be improved separately or replaced by other techniques effective. This can be improved by providing word stream algorithm for voice search in the future work. Further it improves the images retrieval from the pool and reduces the manual work. The query terms are enhanced through the addition of its most probable alternatives to improve retrieval effectiveness.

\section{References}

[1] Xiaoou Tang, Fang Wen "IntentSearch: Capturing User Intention for One-Click Internet Image Search" IEEE Transactions on Pattern Analysis And Machine Intelligence, Vol. 34, No. 7, July 2012.

[2] Amandeep Khokher, Rajneesh Talwar "Content-based Image Retrieval: Feature Extraction Techniques and Applications" International Conference on Recent Advances and Future Trends in Information Technology (iRAFIT2012).

[3] Blaser, A. 1979. Database Techniques for Pictorial Applications, Lecture Notes in Computer Science, Springer Verlag GmbH.

[4] Michele Merler and Rong Yan, John R. Smith "Imbalanced RankBoost for Efficiently Ranking Large-Scale Image/Video Collections".

[5] Gal Chechik and Varun Sharma "Large Scale Online Learning of Image Similarity Through Ranking" Journal of Machine Learning Research 11 (2010) 1109-1135.

[6] J. Cui, F. Wen, and X. Tang, "Real Time Google and Live Image Search Re Ranking," Proc. 16th ACM Int'l Conf. Multimedia, 2008.

[7] J. Cui, F. Wen, and X. Tang, "IntentSearch: Interactive On-Line Image Search Re-Ranking," Proc. 16th ACM Int'l Conf. Multimedia, 2008. 


\section{Bibliography}

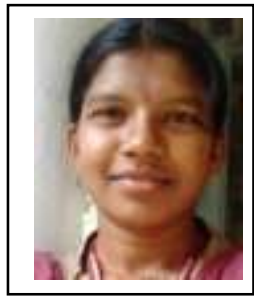

Mrs. M.Veena Sharanya received her B.TECH IT in Sri Shakthi Institute of Engineering and Technology, Coimbatore and she is now pursuing her ME. in Computer Science \& Engineering at Bannari Amman Institute of Technology, Sathyamangalam, Erode, Tamil Nadu, India. She had participated in Various National Level Technical Symposium held at Engineering Colleges in Tamil Nadu and got many Prizes. She had published 2 Papers in Various National and International Conferences held at Engineering Colleges. She had attended more than 5 workshops and seminars in diverse disciplines held at various Engineering Colleges.

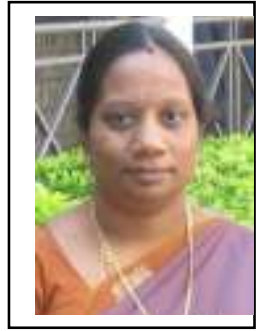

Prof. V. Evelyn Brindha received her M.E in Computer Science \& Engineering and is now pursuing her Ph.D. in Digital Image Processing at Anna University, Chennai. Currently, she is working as Associate Professor in the Department Computer Science and Engineering, Bannari Amman Institute of Technology, Sathyamangalam, Erode, Tamil Nadu, and India. She has 11 years of experience in teaching field. She published five papers in International journals. She presented 4 papers in International Conferences and 4 papers in various National Conferences held at various reputed Engineering colleges. 